Journal of Applied AnALysis

Vol. 8, No. 1 (2002), pp. 1-21

\title{
THE INFINITE DIMENSIONAL HENSTOCK INTEGRAL AND PROBLEMS OF BLACK-SCHOLES EXPECTATION
}

\author{
P. MULDOWNEY
}

Received January 19, 2001 and, in revised form, June 29, 2002

\begin{abstract}
Using arguments which apply equally well to the study of Brownian motion and Feynman path integrals, the relationship between two expressions which arise in derivative asset pricing theory is examined. Detailed explanations are given for some of the key points in the theory of Henstock integrals in function spaces.
\end{abstract}

\section{Introduction}

Suppose the price or value of a financial security is given by $x(t)$ at times $t$ between $t_{0}$ and $T$. It is assumed in the Black-Scholes theory of option pricing that the random variable $x(t)$ has a lognormal distribution; see $[1,6,11]$. This means that the joint probability, that the price $x\left(t_{j}\right)=x_{j}$ will lie in $I_{j}=\left[u_{j}, v_{j}\left[\right.\right.$ for times $t_{1}, \ldots, t_{n}$, is related to

$$
\int_{u_{1}}^{v_{1}} \cdots \int_{u_{n}}^{v_{n}} \prod_{j=1}^{n}\left\{\frac{\exp \left[-\frac{\left(\ln y_{j}-\ln y_{j-1}\right)^{2}}{2\left(t_{j}-t_{j-1}\right)}\right]}{y_{j}\left(2 \pi\left(t_{j}-t_{j-1}\right)\right)^{1 / 2}} d y_{j}\right\} \text {. }
$$

2000 Mathematics Subject Classification. 28C20, 60H05, 81S40, $91 \mathrm{~B} 28$.

Key words and phrases. Geometric Brownian motion, Feynman path integrals.

ISSN 1425-6908（C) Heldermann Verlag. 
Analogous expressions arise in the study of Brownian motion [9], and in Feynman path integrals in quantum mechanics [10], and they can be handled by the methods of this article. We examine the connection between this expression and the related expression

$$
\prod_{j=1}^{n}\left\{\frac{\exp \left[-\frac{\left(\ln x_{j}-\ln x_{j-1}\right)^{2}}{2\left(t_{j}-t_{j-1}\right)}\right]}{x_{j}\left(2 \pi\left(t_{j}-t_{j-1}\right)\right)^{1 / 2}}\left|I_{j}\right|\right\},
$$

where $\left|I_{j}\right|:=v_{j}-u_{j}, j=1, \ldots, n$. Either of these expressions can be regarded as some function $p$ of sets $I$ where

$$
I=\left\{x: x\left(t_{j}\right) \in I_{j}, \quad 1 \leq j \leq n\right\} .
$$

In finance, and in the other subjects mentioned above, we are often concerned with determining the expectation $E^{p}(f)$, relative to probabilities $p$, of certain functions $f$ of the random variables $\left\{x(t): t_{0}<t \leq T\right\}$.

This in effect requires us to be able to integrate $f$ with respect to $p$ in the sample space for the random process $x$, namely the space of functions $x(t)$, $t_{0} \leq t \leq T$. (The parameter $x\left(t_{0}\right)$ may be considered to be variable in the Black-Scholes theory, but is not considered to be random.) This sets the scene for the Henstock integral in this infinite-dimensional function space.

\section{Generalised Riemann integration}

We start by reviewing the Henstock integral in one dimension and in $n$ dimensions. Let $I$ be a real interval of the form

$$
]-\infty, v], \quad[u, v[, \quad \text { or }[u, \infty[,
$$

and let $|I|$ denote $0, v-u$, or 0 respectively. Let $\delta(x)$ be a positive function defined for $x \in \overline{\mathbb{R}}=\mathbb{R} \cup\{-\infty, \infty\}$. The function $\delta$ is called a gauge. We say that $I$ is attached to $x$ (or associated with $x$ ) if

$$
x=-\infty, \quad x=u \text { or } v, x=\infty
$$

respectively. If $I$ is attached to $x$ we say that $(x, I)$ is $\delta$-fine if

$$
v<-\frac{1}{\delta(x)}, \quad v-u<\delta(x), \quad u>\frac{1}{\delta(x)}
$$

respectively. An elementary set $E$ is an interval or a finite union of intervals. $\mathbb{R}$ is an elementary set. A finite collection of attached point interval pairs

$$
\mathcal{E}=\{(x, I)\}=\left\{\left(x^{(1)}, I^{(1)}\right), \ldots,\left(x^{(m)}, I^{(m)}\right)\right\}
$$

is a division of $E$ if the $I^{(j)}$ are disjoint and have union $E$. The division $\mathcal{E}$ is $\delta$-fine, or is a $\delta$-division, if each $(x, I)$ of the division is $\delta$-fine. In that case we write the division as $\mathcal{E}_{\delta}$. 
Suppose $h$ is a real- or complex-valued function of attached point-interval pairs $(x, I)$, with $h(x, I):=0$ at points $x$ for which $h$ may be undefined such as $x= \pm \infty$. For example, $h(x, I)$ could be a point function $f(x)$ multiplied by the interval length $|I|=v-u$, or by some other function of $I$. We say that $h$ is (generalised Riemann) integrable in $E$, with integral $\alpha$, if for every $\varepsilon>0$ there exists a gauge $\delta$ such that

$$
\left|\left(\mathcal{E}_{\delta}\right) \sum h(x, I)-\alpha\right|<\varepsilon
$$

for every $\delta$-division $\mathcal{E}_{\delta}$ of $E$. Write $\alpha=\int_{E} h(x, I)$.

Financial asset prices take only positive values, so we address the question of integration in such spaces. If $\mathbb{R}_{+}$denotes the subset of positive numbers in $\mathbb{R}$, to perform integration in $\mathbb{R}_{+}$we only have to amend the above definitions as follows. Intervals $I=] 0, v[$ have $|I|:=0$; such an $I$ can only be attached to $x=0$; and then $(0, I)$ is $\delta$-fine if $v<\delta(0)$. Also integrand values at $x=0$ (as well as at $x=\infty$ ) are always taken to be zero in the space $\mathbb{R}_{+}$.

For integration in $\mathbb{R}^{n}$ (or $\mathbb{R}_{+}^{n}$ ), intervals $I$ are

$$
I=I_{1} \times \cdots \times I_{n}
$$

where each $I_{j}$ is a one-dimensional interval. $I$ is attached to $x=\left(x_{1}, \ldots, x_{n}\right)$ in $\mathbb{R}^{n}$ (or $\mathbb{R}_{+}^{n}$ ) if each $I_{j}$ is attached to $x_{j}$ in $\mathbb{R}\left(\right.$ or $\mathbb{R}_{+}$). If a gauge $\delta$ is defined in the $n$-dimensional space, $\delta: \mathbb{R}^{n} \mapsto \mathbb{R}_{+}$(or $\mathbb{R}_{+}^{n} \mapsto \mathbb{R}_{+}$), an associated pair $(x, I)$ is $\delta$-fine provided each $\left(x_{j}, I_{j}\right)$ is $\delta$-fine in one-dimensional space. For bounded intervals $I$ with $I_{j}=\left[u_{j}, v_{j}[, j=1, \ldots, n\right.$, this means that each $v_{j}-u_{j}<\delta(x)$. Provided we give the appropriate $n$-dimensional meaning to each of the parameters, the above definition of the integral serves also to define the integral in $n$-dimensional space of a function $h$ of attached interval-point pairs.

\section{The Henstock integral in function space}

If $x$ is a member of a family $C$ of functions defined on a space $B$ and taking values in a space $Y$, then

$$
C \subseteq Y^{B}
$$

For instance, $B$ could be $\left.] t_{0}, T\right], Y$ could be $\mathbb{R}_{+}$, and $C$ could be the family of positive-valued continuous functions on $\left.] t_{0}, T\right]$. If $f$ is a functional of $x \in C$, we may, as discussed in the Introduction above, be interested in the expectation

$$
E^{p}(f)=\int_{C} f(x) d p
$$

where the latter integration has been given some meaning and definition. 
In Henstock integration we tackle this problem by defining the integral in the Cartesian product space

$$
\mathbb{R}_{+}^{B}=\prod_{t \in B} \mathbb{R}_{+}=\left\{x: x(t) \in \mathbb{R}_{+} \text {for } t \in B=\left[t_{0}, T\right]\right\},
$$

so

$$
E^{p}(f)=\int_{\mathbb{R}_{+}^{B}} \mathbf{1}_{C}(x) f(x) d p
$$

whenever the latter integral exists. $\left(\mathbf{1}_{C}\right.$ is the characteristic function or indicator function for $C$.) If $x$ is not restricted to positive values but can take any real (or complex) values, replace $\mathbb{R}_{+}$by $\mathbb{R}$ (or by the space $\mathbf{C}$ of complex numbers) above.

We will define gauge and integral in $\mathbb{R}^{B}$. (As in the finite-dimensional cases, it is easy to adapt the definitions to $\mathbb{R}_{+}^{B}$. In the Black-Scholes application we take $B$ to be $\left.] t_{0}, T\right]$. But in the definitions which follow, $B$ can be taken to be any labelling set. $B$ may have an infinite number of elements, but if it happens to be finite, the definitions reduce naturally to the those relevant to the Henstock integral in finite-dimensional spaces.)

The integral will be defined by Riemann sums $\sum f(x) p(I)$ where the sets $I$ are intervals or cylindrical intervals in $\mathbb{R}^{B}$. If $N$ is a finite subset $\left\{t_{1}, \ldots, t_{n}\right\}$ of $B$, a cylindrical interval $I$ or $I[N]$ is

$$
\prod_{j=1}^{n} I_{j} \times \mathbb{R}^{B \backslash N}
$$

where each $I_{j}$ is a one-dimensional interval. We write $\prod_{j=1}^{n} I_{j}$ as $I(N)$ and $\left(x_{1}, \ldots, x_{n}\right)$ as $x(N)$. So

$$
I=I[N]=\{x: x(N) \in I(N)\} .
$$

We say that $x, N$ and $I[N]$ are attached (or associated) if $I_{j}$ is attached to $x_{j}$ for $1 \leq j \leq n$. The Riemann sums for the function space integrals will be $\sum f(x) p(I)$, or more generally,

$$
\sum h(x, N, I)
$$

where the $x, N, I$ are attached. $h$ may depend explicitly on the dimension labels $t_{j}$ in $N$. Also, $h(x, N, I)$ will be zero by definition if $x_{j}$ is $\pm \infty$ for any $t_{j} \in N$ (or, in the case of the integral in $\mathbb{R}_{+}^{B}$, if $x_{j}$ is 0 or $\infty$ ). The integral will exist if the Riemann sums converge, in some sense, to a single value. So we need some "shrinking rule" or gauge for the associated $I, N$, $x$, which appear in the terms of the Riemann sums. The ideas involved can be illustrated by means of diagrams.

Examples of cylindrical intervals, and the points with which they may be associated in Riemann sums, are shown in Figure 1. For instance, $I$ is 
shown to be restricted in two dimensions $\left(t_{1}\right.$ and $\left.t_{2}\right)$, so the finite set $N$ of $I=I[N]$ may be $\left\{t_{1}, t_{2}\right\}$, and the diagram shows $I$ to be unrestricted in another dimension. Figure 1 tells us nothing about the character of $I$ in the uncountably infinitely many dimensions which are not portrayed.

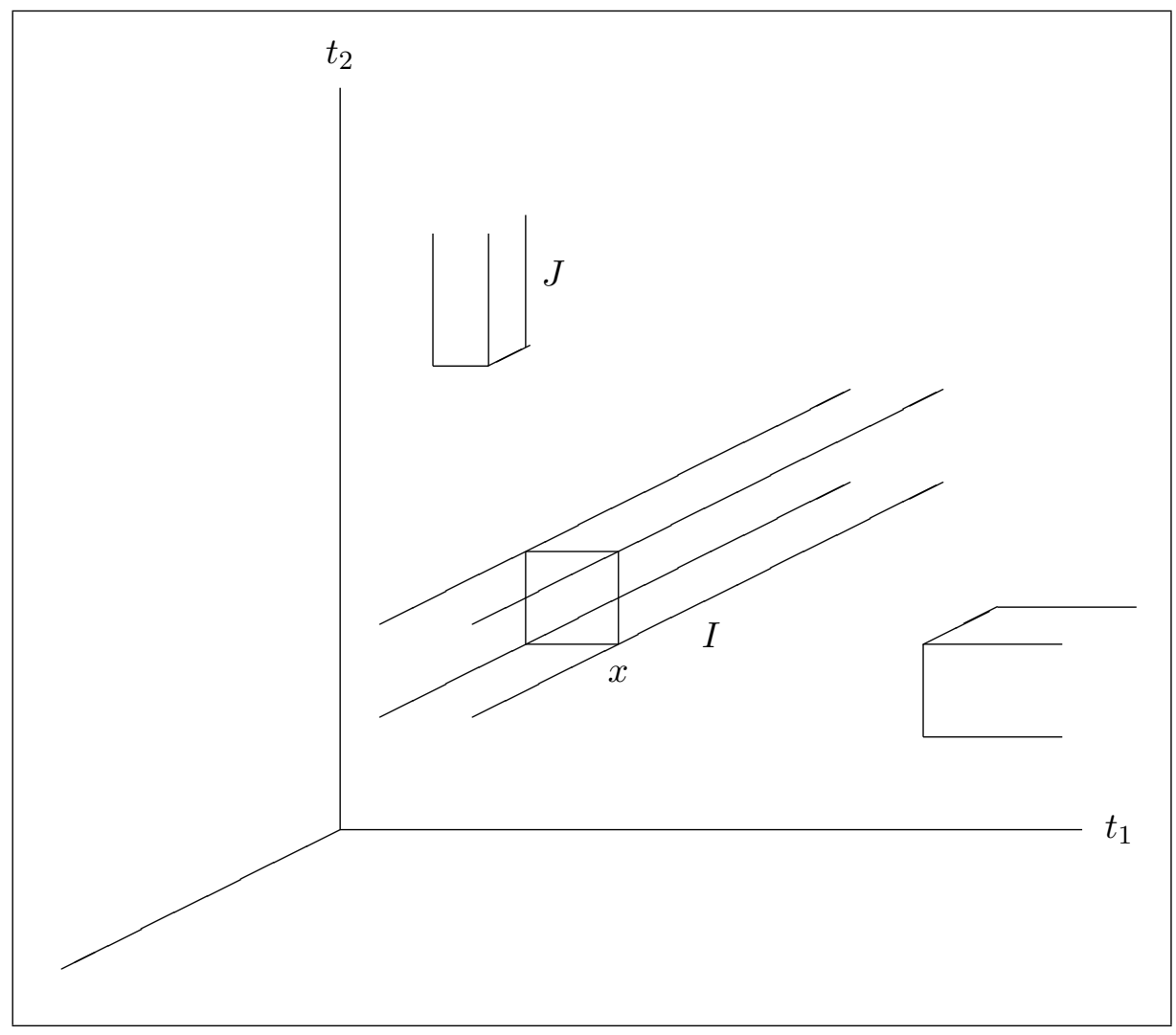

FiguRE 1.

The cylindrical set $I$ may be taken to be attached to, or associated with, the illustrated point $x$; with $x$ lying somewhere in an edge of $I$ in the Cartesian representation; so $\left(x_{1}, x_{2}\right)=\left(x\left(t_{1}\right), x\left(t_{2}\right)\right)$ is a vertex of the twodimensional cell, illustrated in Figure 1, into which $I$ projects in dimensions $t_{1}$ and $t_{2}$.

The cylindrical interval $J$ of Figure 1 is restricted but unbounded in dimension $t_{2}$. In a Riemann sum, such a set can only be attached to a point $y$ which has $y\left(t_{2}\right)=\infty$, and the function value of such a term in a Riemann sum is zero by definition. To form a Riemann sum, we must be able to cover exhaustively the domain of integration with cylindrical intervals such as those appearing in Figure 1. Much of this paper is concerned with proving that such covers can be constructed in certain ways. 
If a function is integrable we expect the Riemann sums to converge, in some sense, to some unique value. We expect the convergence to involve successive (in some sense) Riemann sums whose terms have successively smaller (in some sense) cylindrical sets $I$.

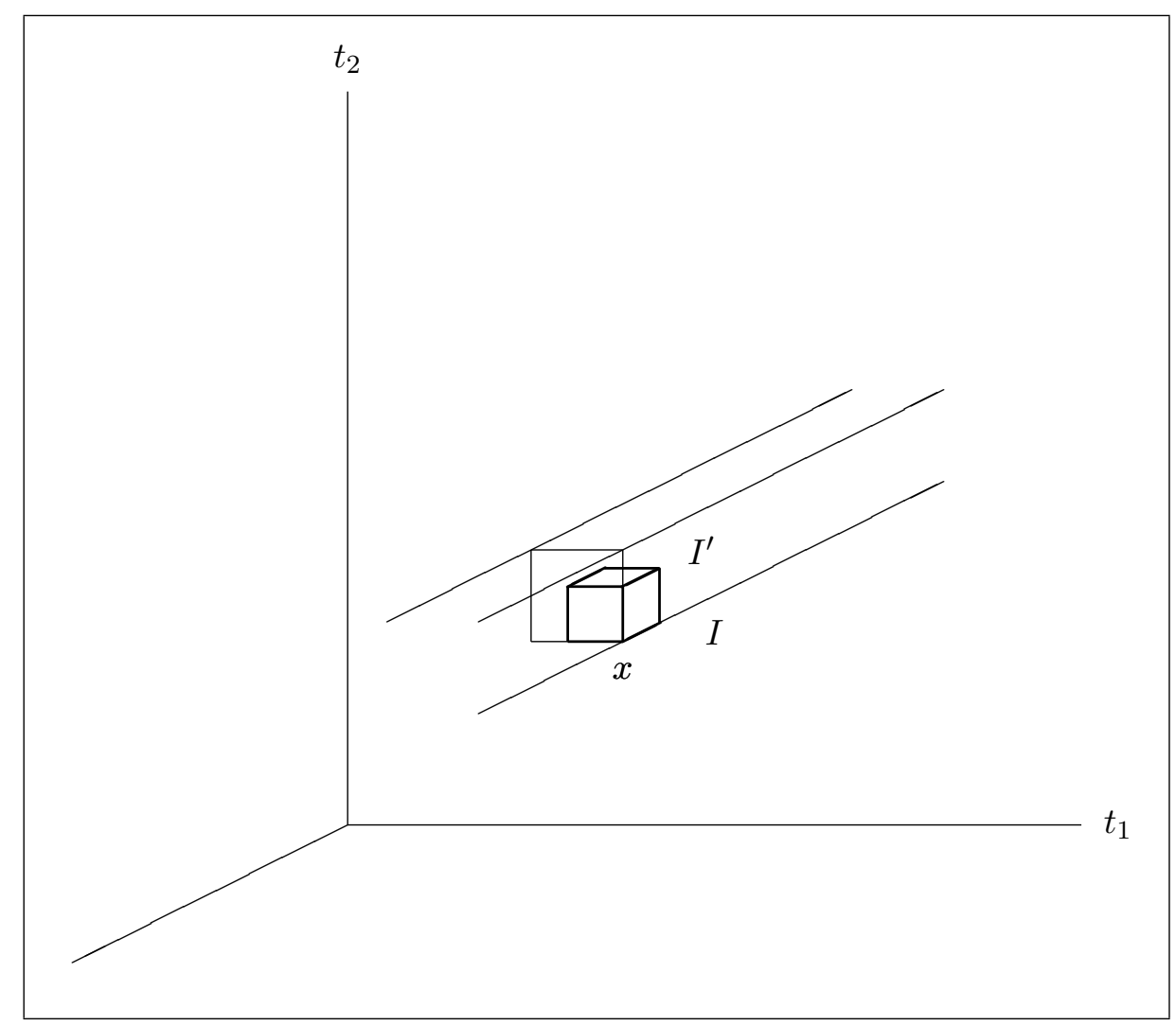

FiguRE 2.

Figure 2 shows how this may be achieved. The set $I$ in Figure 2 is intended to be the same set $I$ of Figure 1, and contains the proper subset $I^{\prime}$ marked with thick lines in Figure 2. The set $I^{\prime}$ includes, among its restricted dimensions, $t_{1}$ and $t_{2}$ which were already restricted in $I$. The edges of $I^{\prime}$ in each of these dimensions are shorter than the corresponding edges of $I$. Furthermore, $I^{\prime}$ is also restricted in a dimension in which $I$ is unrestricted. So a proper subset of a cylindrical set can be obtained by:

- reducing the lengths of the restricted edges (just as we do in finitedimensional integration, using a gauge function $\delta(\cdot))$, and

- increasing the set of restricted dimensions.

We utilise both of these kinds of "shrinkage" in order to define an integration process in infinite-dimensional spaces. 
Firstly, at any point $x$ to which a cylindrical interval is to be attached in a Riemann sum, we specify maximal positive numbers $\delta(x)$ such that the restricted edges of any attached cylindrical set must be shorter than $\delta(x)$. (In fact, as we see below, we need to go a little bit further, and specify maximal lengths in different dimensions $t$, so that this element of the gauge may be different, not only for different points $x$, but also for all of the different restricted dimension sets $N$ into which any such $x$ may be projected.)

Secondly, at each point $x$, we choose a finite, minimal set $L(x)$ of dimensions $t$, so that any cylindrical interval $I=I[N]$, which is attached to $x$ in a Riemann sum, must be such that its finite set $N$ of restricted dimensions contains the minimal set $L(x)$. Just as the lengths of the restricted edges of a cylindrical interval may be arbitrarily small, with upper bound $\delta(x(N))$, the set of restricted dimensions $N$ may be arbitrarily large, "bounded below" by the minimal subset $L(x)$.

The next step is to make these ideas more precise.

\section{Definition of gauges}

There are several ways in which we can have some rule (or gauge) for determining whether or not we have convergence of the Riemann sum approximations to the integral in $\mathbb{R}^{B}$, and we now examine some of these different types of gauges.

Let $A$ be a countable subset of $B$. Let $\mathbf{L}_{A}$ denote the family of all finite subsets of $A$, and let $\mathbf{N}$ denote the family of all finite subsets of $B$. Let $L_{A}$ be a mapping from $\overline{\mathbb{R}}^{B}$ to $\mathbf{L}_{A}$, and let $L$ be a mapping from $\overline{\mathbb{R}}^{B}$ to $\mathbf{N}$,

$$
L_{A}: \overline{\mathbb{R}}^{B} \mapsto \mathbf{L}_{A}, \quad L: \overline{\mathbb{R}}^{B} \mapsto \mathbf{N},
$$

so that each $L_{A}(x)$ is a finite subset of $A$, and each $L(x)$ is a finite subset of $B$. Thus $L_{A}(x)$ and $L(x)$ denote two different ways in which each $x \in \overline{\mathbb{R}}^{B}$ is allocated its own finite set of dimensions determining the minimal dimension sets of intervals $I$ which may be attached to $x$ in Riemann sums. Consider the following positive-valued functions, each defined on a different kind of domain:

$$
\delta: \overline{\mathbb{R}}^{B} \mapsto \mathbb{R}_{+}, \quad \delta_{t}: \overline{\mathbb{R}}^{\{t\}} \mapsto \mathbb{R}_{+}, \quad \delta_{N}: \overline{\mathbb{R}}^{N} \mapsto \mathbb{R}_{+},
$$

so $\delta(x)>0$ for $x \in \overline{\mathbb{R}}^{B}, \delta_{t}(x(t))>0$ for $x(t) \in \overline{\mathbb{R}}^{\{t\}}$ and $t \in B, \delta_{N}(x(N))>0$ for $x(N) \in \overline{\mathbb{R}}^{N}$ and $N \in \mathbf{N}$.

Let $\gamma_{1}, \ldots, \gamma_{5}$, respectively, denote the following: $\left(A, L_{A}, \delta\right),\left(A, L_{A},\left\{\delta_{t}\right\}_{t \in B}\right),\left(A, L_{A},\left\{\delta_{N}\right\}_{N \in \mathbf{N}}\right),\left(L,\left\{\delta_{t}\right\}_{t \in B}\right),\left(L,\left\{\delta_{N}\right\}_{N \in \mathbf{N}}\right)$. 
For $i=1, \ldots, 5$, we say that the attached point-interval pair $(x, I[N])$ is $\gamma_{i}$-fine if:

$$
\begin{aligned}
& i=1: N \supseteq L_{A}(x) \text { and }(x(t), I(t)) \text { is } \delta \text {-fine in } \overline{\mathbb{R}}^{\{t\}} \text { for } t \in N, \\
& i=2: N \supseteq L_{A}(x) \text { and }(x(t), I(t)) \text { is } \delta_{t} \text {-fine in } \overline{\mathbb{R}}^{\{t\}} \text { for } t \in N, \\
& i=3: N \supseteq L_{A}(x) \text { and }(x(N), I(N)) \text { is } \delta_{N} \text {-fine in } \overline{\mathbb{R}}^{N}, \\
& i=4: N \supseteq L(x) \text { and }(x(t), I(t)) \text { is } \delta_{t} \text {-fine in } \overline{\mathbb{R}}^{\{t\}} \text { for } t \in N, \\
& i=5: N \supseteq L(x) \text { and }(x(N), I(N)) \text { is } \delta_{N} \text {-fine in } \overline{\mathbb{R}}^{N} .
\end{aligned}
$$

Any finite collection of attached point-interval pairs is $\gamma_{i}$-fine if each member is $\gamma_{i}$-fine.

In the case of gauges of the type $\gamma_{1}$, we can vary each of the parameters $A, L_{A}$ and $\delta$ as follows. The set $A$ will be taken successively "larger"; for each $x, L_{A}(x)$ will be successively "larger"; for each $x, \delta(x)$ will be successively smaller. By this means we will determine whether or not Riemann sum approximations converge to an integral. Likewise for the approaches indicated in $i=2$ to 5 .

A division of $\mathbb{R}^{B}$ is a finite collection $\mathcal{E}=\{(x, I[N])\}$ of attached pointinterval pairs such that the $I[N]$ are disjoint and have union $\mathbb{R}^{B}$. If $\mathcal{E}$ is $\gamma_{i}$-fine (so each $(x, I[N])$ is $\gamma_{i}$-fine) we write it as $\mathcal{E}_{\gamma_{i}}$.

We shall see below (Proposition 1 and Proposition 2) that gauges of the type $\gamma_{1}$ and $\gamma_{2}$ each enables us to define an integration method in $\mathbb{R}^{B}$, because $\gamma_{i}$-fine divisions of $\mathbb{R}^{B}$ exist for $i=1,2$. Gauges $\gamma_{2}$ are "better" than $\gamma_{1}$, in the sense that functions that are integrable in the sense of $\gamma_{2^{-}}$ gauges are also integrable in the sense of $\gamma_{1}$-gauges. It is not known whether gauges of type $\gamma_{3}, \gamma_{4}$ or $\gamma_{5}$ enable us to define an integration in $\mathbb{R}^{B}$. If $\delta_{t}$ is given for $t \in B$, we can define $\delta_{N}$ so that $\delta_{N}(x(N))<\delta_{t}(x(t))$ for $t \in N$; and this would enable us to compare any integration based on $\gamma_{3}$ or $\gamma_{5}$ with $\gamma_{2}$ or $\gamma_{4}$, respectively.

The key point in showing that $\gamma_{i}$ determines an integration is to prove that a $\gamma_{i}$-fine division of $\mathbb{R}^{B}$ exists. Gauges of type $\gamma_{2}$ are described in $[2,3,4]$ and $\gamma_{4}$ in [5]. Gauges $\gamma_{1}$ are described, with proof, in [8]; the proof is repeated below (Proposition 1). A proof for $\gamma_{2}$ is also given below (Proposition 2). No correct proofs for gauges of the types $\gamma_{3}, \gamma_{4}$ or $\gamma_{5}$ have yet been given.

\section{Aspects of gauge}

To illustrate the issues involved in gauges for Riemann-type integration in $\mathbb{R}^{B}$, we now examine various features of gauges of type $\gamma_{3}$. 
The sets $L_{A}(x)$ are finite, but as we look at successive choices of gauge $\gamma_{3}$ we can make these sets successively larger. This ensures an increasing number of dimensions $t$ in the sets $N$ of restricted dimensions of the $\gamma_{3}$-fine cylinder sets $I[N]$. In other words, these cylinder sets have an increasing number of bounded or finite edges, corresponding to the successive choices of gauge $\gamma_{3}$. Notice also that while the $N$ of any $I[N]$ attached to $x$ must contain the subset $L_{A}(x)$ of $A$, the set $N$ itself need not be a subset of $A$.

The other factor in $\gamma_{3}$ is the finite-dimensional gauges $\delta_{N}$. As we consider successive gauges $\gamma_{3}$, we can choose the $\delta_{N}$ so that the restricted edges of $\gamma_{3}$-fine $I[N]$ are successively shorter, corresponding to the successive gauges.

Thus we can choose successive gauges $\gamma_{3}$ so that $\gamma_{3}$-fine cylinder sets $I[N]$ have, successively a greater number of restricted edges, and/or, successively shorter restricted edges. In other words, the cylinder sets $I$ are forced to be successively smaller in some sense. But this is exactly the role and purpose of the more familiar Henstock gauges in one dimension.

So where does the countable dimension set $A$ come into all this? One of the reasons for having $A$ in the definition will emerge whenever we go on to prove that $\gamma_{1}$-fine and $\gamma_{2}$-fine divisions exist. We do not know how to prove this without including $A$ in the definition.

To recap, a particular gauge $\gamma_{3}$ is determined by a particular countable set $A$, a particular mapping $L_{A}$, and a family $\left\{\delta_{N}\right\}_{N \in \mathbf{N}}$ of particular gauges $\delta_{N}$ in $\mathbb{R}^{N}$.

The gauges $\gamma_{3}$ give a "shrinking rule" for the cylinder sets $I[N]$ which are attached to points $x$, at which an integrand $f(x)$ is evaluated, in Riemann sums $\sum f(x) p(I[N]), p$ being some integrator function, or volume, defined on the cylinder sets.

The reader is invited to speculate on the kinds of integration that may emerge from alternative definitions of gauge. One basic test is whether the kind of gauge used enables reasonably well-behaved cylinder functions to be integrated. (A cylinder function is a functional defined for $x$ in $\mathbb{R}^{B}$, but whose values depend only on the values assumed by $x$ at a fixed finite subset of dimensions in $B$. See [7], Proposition 38, page 56.)

\section{Existence of $\gamma$-divisions}

We prove first that $\gamma_{1}$-fine divisions exist. This proof was given in [8]. Let $E$ be any elementary set (an interval or a finite union of intervals). Let a gauge $\gamma_{1}$ be given by $\left(A, L_{A}, \delta\right)$. The following result is proved in the space $\mathbb{R}^{B}$, but is easily adapted for $\mathbb{R}_{+}^{B}$.

Proposition 1. There exists a $\gamma_{1}$-division of $E$. 
Proof. We show that there exists a $\gamma_{1}$-fine division of $\mathbb{R}^{B}$. The proof can easily be amended with $E$ in place of $\mathbb{R}^{B}$. Suppose such a division does not exist. We will deduce a contradiction from this assumption. Let $\left\{r_{j}\right\}_{j=1}^{\infty}$ be an enumeration of $A$. Let $q$ be a positive integer. For any $t$, let $I(t)$ denote any of the real intervals $]-\infty,-q\left[, \quad\left[s 2^{-q},(s+1) 2^{-q}[, \quad[q, \infty[\right.\right.$, where $s$ is any integer satisfying $-q 2^{q} \leq s \leq q 2^{q}-1$. As $s$ takes all its permitted values, the resulting collection $\mathcal{I}^{q}$ of mutually disjoint $I(t)$ form a cover for $\mathbb{R}=]-\infty, \infty\left[\right.$. Let the positive integer $m$ be given, and let $A_{m}=\left\{r_{1}, r_{2}, \ldots\right.$, $\left.r_{m}\right\} \subset A$. For given $q, m$, consider the family of $I\left(r_{1}\right) \times I\left(r_{2}\right) \times \cdots \times I\left(r_{m}\right)$, the family being formed by taking every possible choice of $I(t) \in \mathcal{I}^{q}\left(t \in A_{m}\right)$ in the product, and, for any such choice, let $I^{q m}$ denote the cylindrical interval

$$
I\left(r_{1}\right) \times I\left(r_{2}\right) \times \cdots \times I\left(r_{m}\right) \times \prod\left\{\mathbb{R}: t \in B \backslash A_{m}\right\} .
$$

The resulting family of mutually disjoint cylindrical intervals $I^{q m}$, with $q$, $m$ fixed, covers $\mathbb{R}^{B}$. Since, by assumption, there does not exist a $\gamma_{1}$-division of $\mathbb{R}^{B}$, then, for each $q, m$, there exists $I^{q m}$ for which there is no $\gamma_{1}$-fine division. (Because if there is a $\gamma_{1}$-division for every $I^{q m}$, their union (finite) is a $\gamma_{1}$-division for $\mathbb{R}^{B}$ ). We now show that, on the assumption that no $\gamma_{1}$-division of $\mathbb{R}^{B}$ exists, we can find a descending sequence

$$
I^{11} \supset \cdots \supset I^{q q} \supset I^{q+1, q+1} \supset \cdots
$$

for each of which there is no $\gamma_{1}$-division. They are found, by induction, as follows. The original assumption starts off the inductive chain. Now suppose that $I^{q q}$ has no $\gamma_{1}$-division. Choose $I^{q+1, q} \subset I^{q q}$ for which there is no $\gamma_{1}$-division. There must be such a set, because if there were not, then the $\gamma_{1}$-fine divisions of each of the finite number of $I^{q+1, q}$ would combine to give a $\gamma_{1}$-division of $I^{q q}$. Then, by the same reasoning, choose $I^{q+1, q+1} \subset I^{q+1, q}$ for which there is no $\gamma_{1}$-division. Thus the inductive chain is established, and we have the sequence (1) above, for which there are no $\gamma_{1}$-fine divisions. Using the Cartesian product topology in $\mathbb{R}^{B}$, and denoting by $\bar{J}$ the closure of $J$ in that topology, the finite intersection property implies there exists $x$ in $\bigcap_{q=1}^{\infty} \bar{I}^{q q}$. Let $k=\max \left\{j: r_{j} \in L_{A}(x)\right\}$. Choose $q^{\prime}>k$ so that $2^{-q^{\prime}}<\delta(x)$. Let $q=\max \left\{k, q^{\prime}\right\}$. Let $N=\left\{r_{1}, r_{2}, \ldots, r_{q}\right\}$. For $t \in N$ let $J(t)$ denote either of the intervals

$$
\left[x(t)-2^{-q}, x(t)\left[, \quad\left[x(t), x(t)+2^{-q}[.\right.\right.\right.
$$

Let $J(N)$ denote any of the $2^{q} q$-dimensional intervals $\prod_{t \in N} J(t)$. So $J[N]$ denotes any of the $2^{q}$ corresponding cylindrical intervals. Each of $(x, J[N])$ is $\gamma_{1}$-fine, as is each of $\left(x, J[N] \cap I^{q q}\right)$. The latter collection is a $\gamma_{1}$-fine division of $I^{q q}$, contradicting the original assumption. This completes the proof. 
Note that, by including intervals of the form $]-\infty,-q[,[q, \infty[$, the collection $\mathcal{I}=\bigcup_{q=1}^{\infty} \mathcal{I}^{q}$ generates $\gamma_{1}$-fine cylinder sets $I[N]$ which have associated points $x$ for which $x(N)$ has one or more infinite components. Such an $x$ may emerge at $\bigcap_{q=1}^{\infty} \bar{I}^{q q}$ above, and that case requires a slightly different version of the final steps of the proof.

It might seem that the above proof would also be valid for gauges of type $\gamma_{3}$. Replacing $\gamma_{1}$ by $\gamma_{3}$ throughout, and continuing from stage $\bigcap_{q=1}^{\infty} \bar{I}^{q q}$ above, we might argue as follows. Let $k=\max \left\{j: r_{j} \in L_{A}(x)\right\}$. Let $N=\left\{r_{1}, \ldots r_{k}\right\}$. Suppose $x(N)$ does not have any infinite component. Choose $q^{\prime}>k$ so that $2^{-q^{\prime}}<\delta_{N}(x(N))$. For $t \in N$ let $J(t)$ denote either of the intervals $\left[x(t)-2^{-q^{\prime}}, x(t)\left[, \quad\left[x(t), x(t)+2^{-q^{\prime}}[\right.\right.\right.$. Let $J(N)$ denote any of the $2^{k} k$-dimensional intervals $\prod_{t \in N} J(t)$. So $J[N]$ denotes any of the $2^{k}$ corresponding cylindrical intervals. Each of $(x, J[N])$ is $\gamma$-fine, as is each of $\left(x, J[N] \cap I^{q^{\prime} q^{\prime}}\right)$. At first sight, the latter collection might seem to be a $\gamma_{3}$-fine division of $I^{q^{\prime} q^{\prime}}$, giving a contradiction as before. But the interval $J[N] \cap I^{q^{\prime} q^{\prime}}$ is restricted, not just in the dimensions $N=\left\{r_{1}, \ldots, r_{k}\right\}$, but in $M=\left\{r_{1}, \ldots, r_{k}, \ldots, r_{q^{\prime}}\right\}$, and $\left(x, J[N] \cap I^{q^{\prime} q^{\prime}}\right)$ may fail to be $\gamma_{3}$-fine, so this approach fails for $\gamma_{3}$.

In the following, recall that a gauge $\gamma_{2}$ is given by $\left(A, L_{A},\left\{\delta_{t}\right\}_{t \in B}\right)$ with $A=\left\{r_{j}\right\}_{j=1}^{\infty} \subset B$.

Proposition 2. There exists a $\gamma_{2}$-division of $\mathbb{R}^{B}$.

Proof. Assume that $\mathbb{R}^{B}$ is not $\gamma_{2}$-divisible. Let $A_{k}=\left\{r_{1}, r_{2}, \ldots, r_{k}\right\}$. Choose a $\delta_{r_{1}}$-fine division $\left\{\left(x\left(r_{1}\right), I\left(r_{1}\right)\right)\right\}$ of $\mathbb{R}^{\left\{r_{1}\right\}}$. Consider each of the finite number of $I\left[\left\{r_{1}\right\}\right]=I\left(r_{1}\right) \times \mathbb{R}^{B \backslash\left\{r_{1}\right\}}$. By hypothesis, at least one of these must be non- $\gamma_{2}$-divisible; select one such and call it $I^{1}\left[A_{1}\right]$. Denote the corresponding $x\left(r_{1}\right)$ by $x^{1}\left(r_{1}\right)$. Proceeding inductively, we suppose $x^{j}\left(r_{j}\right)$ and $I^{j}\left[A_{j}\right]$ have been determined. Consider a $\delta_{r_{j+1}}$-division $\left\{\left(x\left(r_{j+1}\right), I\left(r_{j+1}\right)\right)\right\}$ of $\mathbb{R}^{\left\{r_{j+1}\right\}}$. By hypothesis, there must be at least one $I\left(r_{j+1}\right)$ with $I\left[A_{j+1}\right]=I^{j}\left(A_{j}\right) \times I\left(r_{j+1}\right) \times \mathbb{R}^{B \backslash A_{j+1}}$ non- $\gamma_{2}$-divisible. Select one and write $I\left[A_{j+1}\right]=I^{j+1}\left[A_{j+1}\right]$. Denote the corresponding $x\left(r_{j+1}\right)$ by $x^{j+1}\left(r_{j+1}\right)$. By induction, for $j=1,2,3, \ldots$ we have $\left(x^{j}\left(r_{j}\right), I^{j}\left(r_{j}\right)\right) \delta_{r_{j}}$-fine and $I^{j}\left[A_{j}\right]$ non- $\gamma_{2}$-divisible. Choose $x \in \overline{\mathbb{R}}^{B}$ so that $x\left(r_{j}\right)=x^{j}\left(r_{j}\right)$ for each $r_{j} \in A$, with $x(t)$ arbitrary for $t \in B \backslash A$. For this $x$ choose $k$ so that $L_{A}(x) \subseteq A_{k}$. Since $\left(x^{j}\left(r_{j}\right), I^{j}\left(r_{j}\right)\right)$ is $\delta_{r_{j}}$-fine for $1 \leq j \leq k,\left(x, I^{k}\left[A_{k}\right]\right)$ is a $\gamma_{2}$-fine division of $I^{k}\left[A_{k}\right]$, giving a contradiction. The result follows. The argument can easily be adapted to prove the $\gamma_{2}$-divisibility of any elementary set $E$ in $\mathbb{R}^{B}$. 


\section{Problems of proving $\gamma$-divisibility}

An earlier argument along the lines of Proposition 1 was given in [7]. An error in this was pointed out by Dr. Christian Ott in a letter dated 17/8/92. The error relates to the step where we obtain $I^{q+1, q+1}$ from $I^{q q}$ in (1) above. In [7], Proposition 1 (page 20, line 17) says, "We can take $J^{(q+1, m)} \subseteq J^{(q m)}$ and $J^{(q, m+1)} \subseteq J^{(q m)}$ ". This is false. (These $J^{(q m)}$ 's are, in the notation of Proposition $1, I^{q m}$ 's for which there are no $\gamma_{1}$-divisions.) It may be useful to discuss this point in detail.

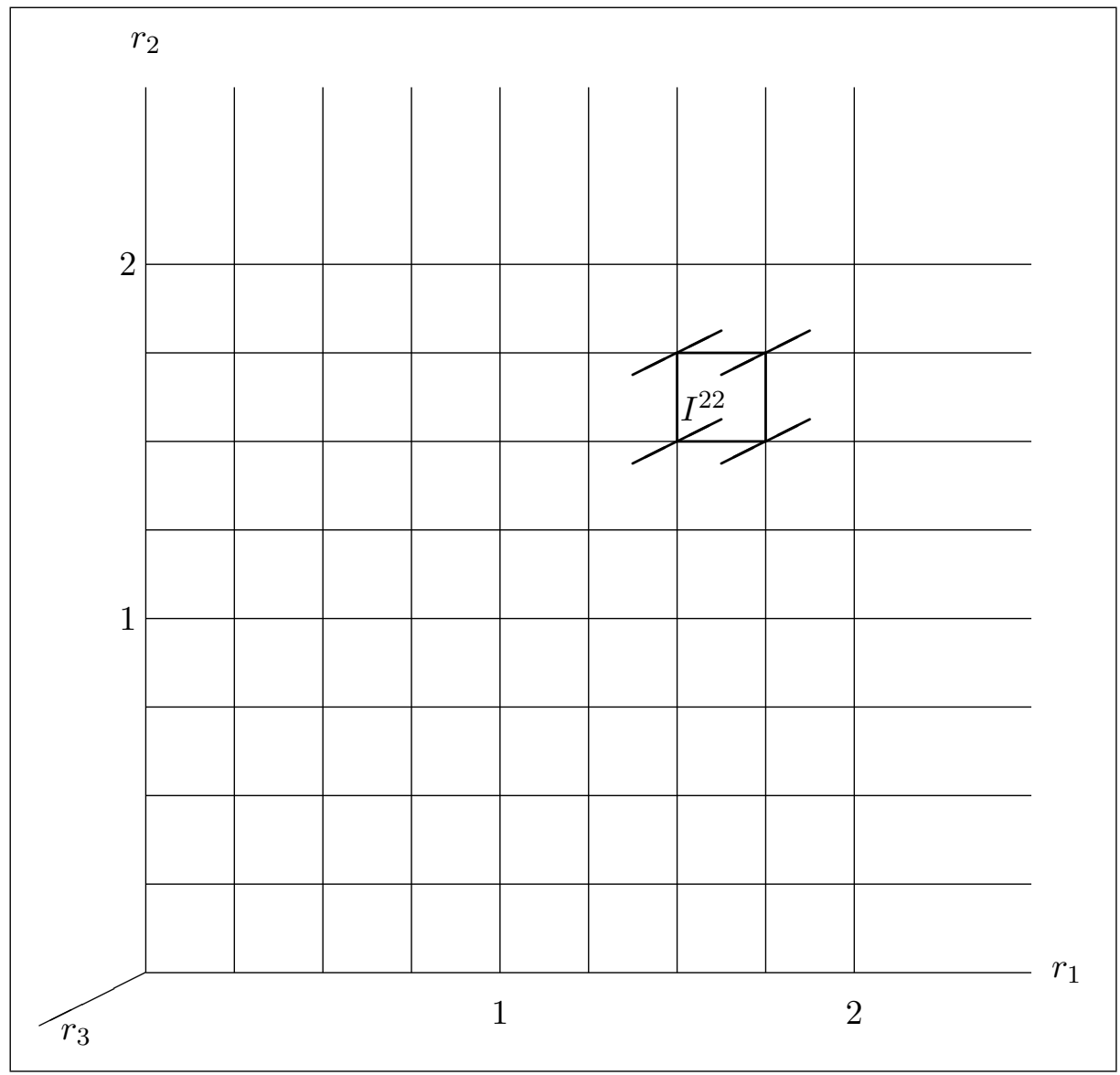

FIGURE 3.

To illustrate the point, let us take $q=2$ and $m=2$. We use somewhat more metaphorical descriptions in an attempt to assist visualisation. Imagine that the dimensions $r_{1}$ and $r_{2}$ are the two dimensions of the surface or page on which these words are written, and that $r_{3}$ is a dimension perpendicular to the page. There are infinitely many other dimensions involved, 
but these are less easy to visualise directly. The flat two-dimensional surface is now covered by a grid, of which that part of the surface bounded by the straight lines joining the four points $( \pm 2, \pm 2)$ consists of squares, or $\left(r_{1}, r_{2}\right)$ cells, of side $1 / 4$. Outside of this area, the surface is divided into 64 regions which are unbounded in one dimension, $r_{1}$ or $r_{2}$; and 4 regions which are unbounded in two dimensions, $r_{1}$ and $r_{2}$. This grid is the 2-dimensional projection of a honeycomb structure for the whole (infinite-dimensional) space; we can easily visualise three dimensions of this honeycomb, with each cell of the honeycomb consisting of an infinitely long (from $-\infty$ to $\infty$ in all of the dimensions other than $r_{1}$ and $r_{2}$ ) shaft passing through each little $1 / 4 \times 1 / 4$ section of the flat two-dimensional grid that we have just considered. Similarly for those sections of the grid which are partly unbounded.

In Figure 3, the set outlined in heavy lines, call it $I^{22}$, is such a shaft.

These shafts are cylindrical intervals. As part of the induction in the proof above, we assumed that at least one of these shafts had no $\gamma_{1}$-fine division. We selected one such. Suppose it is $I^{22}$.

We now attempt the inductive step. When we increase $q$ from 2 to 3 , we refine the grid on the flat surface in dimensions $r_{1}$ and $r_{2}$, so that each of the bounded cells is divided into 4 square cells of side $1 / 8$; and the region covered by the bounded cells now covers the square with vertices $( \pm 3, \pm 3)$.

The honeycomb structure in the infinite-dimensional space, consisting of cylinder sets or shafts, changes correspondingly, so that each of the shafts based on bounded cells in the surface divides into four smaller shafts, each one again stretching from $-\infty$ to $\infty$ in dimension $r_{3}$. In Figure $4, I^{32}$ is such a shaft. (The shafts with some unbounded edges in the $\left(r_{1}, r_{2}\right)$-surface divide up differently.)

By assumption, at least one of these sub-shafts within $I^{22}$ must fail to have a $\gamma_{1}$-fine division; select one and suppose it is $I^{32}$.

What happens if we increase $m$ from 2 to 3 while leaving $q=2$ ? In this case, each of the shafts based on $(1 / 4 \times 1 / 4)$ cells of the flat grid is divided up into 16 pieces (which, in dimensions $r_{1}, r_{2}$ and $r_{3}$ look like cubes with edges of length 1/4) plus two further pieces, one of which (in dimension $r_{3}$ ) is unbounded above, the other unbounded below. Of course in every dimension other than $r_{1}, r_{2}$ and $r_{3}$, these pieces are infinitely long, from $-\infty$ to $+\infty$. In other words, we now have a different honeycomb structure in the space, so that in dimensions $r_{1}, r_{2}$ and $r_{3}$ the cube bounded by the eight points $( \pm 2, \pm 2, \pm 2)$ is divided up into 512 cubes each with edge of length $1 / 4$. One of these cubes is shown as $I^{23}$ in Figure 4. This development still gives a "shaft" or cylindrical honeycomb structure in the infinite-dimensional space. 


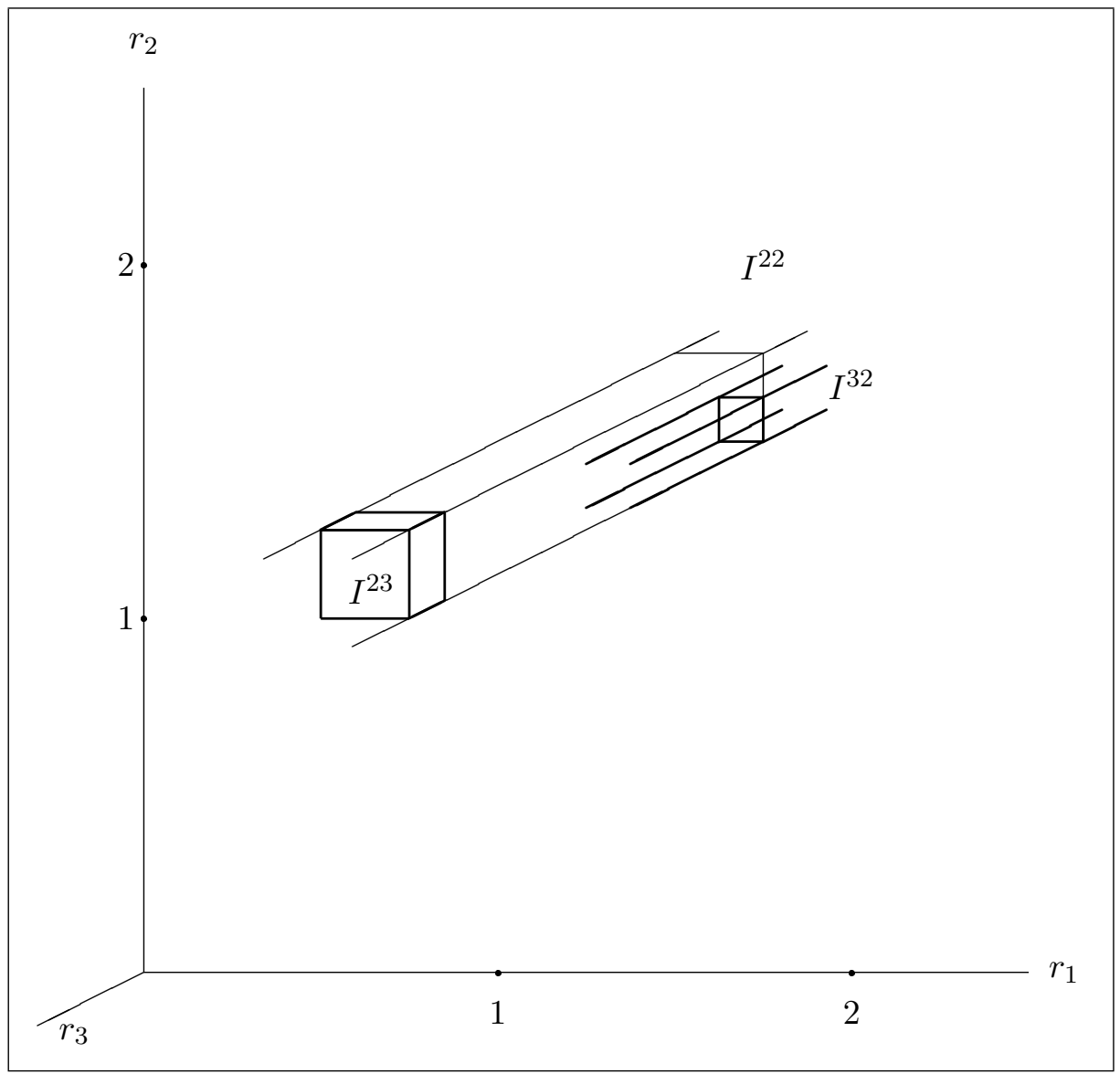

Figure 4.

Returning to $I^{22}$, with $m=3$ this has been divided into 16 pieces, at least one of which, in consequence of our original assumption, does not have a $\gamma_{1}$-fine division; select one such and suppose it is $I^{23}$.

Now $I^{32}$ and $I^{23}$ have a non-empty intersection. So far, so good in the argument of [7]. But the problem here is that there is no guarantee, within the argument given, that this intersection does not have a $\gamma_{1}$-fine division; it may indeed be $\gamma_{1}$-divisible, and this collapses the inductive argument of [7].

We can ensure that the absence of $\gamma_{1}$-divisibility is inherited down the inductive chain. But the reasoning in [7] leads into another difficulty. For if we take each of the $\left(r_{1}, r_{2}, r_{3}\right)$-cells of the non- $\gamma_{1}$-divisible $I^{23}$, each having edges $1 / 4 \times 1 / 4 \times 1 / 4$, and subdivide by bisection, going from $q=2$ to $q=3$, then at least one of the sets arising from this bisection must, by assumption, be non- $\gamma_{1}$-divisible. Select one of these and call it $I^{\prime}$. 
Now consider the non- $\gamma_{1}$-divisible $I^{32}$, which is a shaft or cylinder set, typically bounded in a $1 / 8 \times 1 / 8\left(r_{1}, r_{2}\right)$-cell. Going from $m=2$ to $m=3$, this cylinder is subdivided in dimension $r_{3}$, and one of these subsets must be non- $\gamma_{1}$-divisible; call it $I^{\prime \prime}$.

We have now found $I^{\prime}$ and $I^{\prime \prime}$, both non- $\gamma_{1}$-divisible, and both having $q=3$ and $m=3$; so we seem to have moved through the inductive chain. But a little reflection will show that $I^{\prime}$ and $I^{\prime \prime}$ may fail to intersect, causing the argument of [7] to collapse yet again. It is left to the reader to confirm that the the proof given in Proposition 1 above overcomes these difficulties.

\section{Henstock integral and variation in $\mathbb{R}_{+}^{B}$}

The integrands in the applications described in the Introduction have domain $\mathbb{R}_{+}^{B}$ instead of $\mathbb{R}^{B}$, and the results we have obtained apply equally in this domain.

In this section, we may take the gauge $\gamma$ to be $\gamma_{1}$ or $\gamma_{2}$. Suppose $h$ is a function of attached $x, N, I$. Sometimes $h(x, N, I[N])$ is undefined for certain $x \in \overline{\mathbb{R}}_{+}^{B}$ (or $\overline{\mathbb{R}}^{B}$ ), such as those $x$ for which $x(t)=0$ or $\infty$ for $t \in N$. In such cases we may $h(x, N, I)$ to be zero, and these terms are omitted from the Riemann sum. If $E$ is an elementary set, the variation of $h$ in $E$ is

$$
\inf _{\gamma}\left\{\sup _{\mathcal{E}_{\gamma}}\left\{\left(\mathcal{E}_{\gamma}\right) \sum|h(x, I, N)|\right\}\right\} .
$$

If $X$ is any subset of $\mathbb{R}_{+}^{B}$, the variation of $h$ in $\mathbb{R}_{+}^{B}$ relative to $X$ is

$$
\inf _{\gamma}\left\{\sup _{\mathcal{E}_{\gamma}}\left\{\left(\mathcal{E}_{\gamma}\right) \sum|h(x, I, N)| \mathbf{1}_{X}(x)\right\}\right\}
$$

where $\mathbf{1}_{X}(x)$ is the characteristic function or indicator function of $X$, and the $\mathcal{E}_{\gamma}$ now denote $\gamma$-divisions of $\mathbb{R}_{+}^{B}$. We say that $h$ has bounded variation in $E$ if its variation in $E$ is finite. We say that $h$ is $\mathrm{VBG}^{*}$ (or $h$ has generalised bounded variation in $\mathbb{R}_{+}^{B}$ if $\mathbb{R}_{+}^{B}$ is the union of disjoint sets $X_{j}$ with $h$ having bounded variation in $X_{j}, j=1,2,3, \ldots$.

We say that $h$ is integrable in the elementary set $E$, with integral $\alpha$ denoted by $\int_{E} h(x, N, I)$, if, given $\varepsilon>0$, there exists $\gamma$ so that

$$
\left|\left(\mathcal{E}_{\gamma}\right) \sum h(x, N, I)-\alpha\right|<\varepsilon
$$

for every $\gamma$-fine division $\mathcal{E}_{\gamma}$ of $E$.

For given $\gamma$ let $S_{\gamma}$ denote $\left\{\left(\mathcal{E}_{\gamma}\right) \sum h(x, N, I)\right.$ : all $\left.\mathcal{E}_{\gamma}\right\}$, and let $\bar{S}_{\gamma}$ denote the closure of the set. If $h$ is integrable with $\int_{E} h=\alpha$, then $\{\alpha\}=\bigcap_{\gamma} \bar{S}_{\gamma}$. This can be used as an alternative, equivalent definition of the integral. 
Two functions $h_{1}(x, N, I)$ and $h_{2}(x, N, I)$ are variationally equivalent if $h_{1}-h_{2}$ has variation zero in $\mathbb{R}_{+}^{B}$ ([7], page 32$)$. It is easy to show that $h_{1}$ is variationally equivalent to $h_{2}$ if, given $\varepsilon>0$, there exists $\gamma$ so that, for all $\mathcal{E}_{\gamma}$

$$
\left(\mathcal{E}_{\gamma}\right) \sum\left|h_{1}(x, N, I)-h_{2}(x, N, I)\right|<\varepsilon
$$

If $h_{1}$ is integrable, and if $h_{2}$ is variationally equivalent to $h_{1}$, then $h_{2}$ is integrable and $\int h_{1}=\int h_{2}$. (See [7], Proposition 18, page 32 for proof.)

\section{Applications}

We now consider the integrability of a particular integrand $h$ which arises in the theory of Black-Scholes valuation of stock options [11]. In finance, the positive quantities $r(t)$ and $\sigma$ denote, respectively, the risk-free interest rate and the volatility [11]. Let $r_{j}:=r\left(t_{j}\right)$, and $A_{j}:=\left(2 \pi \sigma^{2}\left(t_{j}-t_{j-1}\right)\right)^{1 / 2}$. Let

$$
\begin{aligned}
g_{j}\left(x_{j}, x_{j-1}\right):=\frac{1}{A_{j} x_{j}} \exp & {\left[-\frac{1}{2 \sigma^{2}}\left\{\frac{\ln x_{j}-\ln x_{j-1}}{t_{j}-t_{j-1}}\right.\right.} \\
& \left.\left.-\left(r_{j}-\frac{1}{2} \sigma\right)\right\}^{2}\left(t_{j}-t_{j-1}\right)\right] .
\end{aligned}
$$

Let

$$
q(I(N)):=\prod_{j=1}^{n} g_{j}\left(x_{j}, x_{j-1}\right)\left|I_{j}\right|,
$$

where $\left|I_{j}\right|=v_{j}-u_{j}$ if $I_{j}$ is $\left[u_{j}, v_{j}[\right.$, and let

$$
Q(I(N)):=\int_{I(N)} \prod_{j=1}^{n} g_{j}\left(y_{j}, y_{j-1}\right) d y(N) .
$$

If $x, N, I$, are associated, let

$$
g(x, N, I):=q(I(N)), \quad G(x, N, I):=Q(I(N)) .
$$

In the following proof, only gauges $\gamma_{2}$ are used, and to highlight this we use the symbol ${ }^{2} \int$ instead of $\int$ in the statement of the result.

Proposition 3. $G(x, N, I)$ is integrable in $\mathbb{R}_{+}^{B}$, and ${ }^{2} \int_{\mathbb{R}_{+}^{B}} G(x, N, I)=1$.

Proof. Essentially this follows from

$$
\int_{\mathbb{R}_{+}} \pi^{-1 / 2} y^{-1} \exp \left[-(\ln y)^{2}\right] d y=1 .
$$


Consider any division $\mathcal{E}$ of $\mathbb{R}_{+}^{B}$, and the Riemann sum

$$
(\mathcal{E}) \sum G(x, N, I),=(\mathcal{E}) \sum Q(I(N)) \text {. }
$$

Let $M=\bigcup\{N:(x, I[N]) \in \mathcal{E}\}$. Enumerate $M$ as $\left\{t_{1}, \ldots, t_{m}\right\}$. Each term $Q(I(N))$ of the Riemann sum can be rewritten as $Q(I(M))$ by inserting additional $y_{j}$ 's in expression $g_{j}$ of (4) and adding an integration from zero to plus infinity on the extra $y_{j}$ 's. Then the Riemann sum (7) becomes

$$
(\mathcal{E}) \sum Q(I(M))
$$

with $M$ a fixed set of dimensions, so we are now dealing, in effect, with some Riemann sum estimate of an integral in $m$ dimensions. Suppose we take the $\operatorname{sum}(\mathcal{E}) \sum G(x, N, I[N])$ to have no terms for which $x(t)$ is zero or $\infty$ for $t \in N$. Let $E:=\bigcup\{I:(x, I[N]) \in \mathcal{E}\}$, and let $F:=\mathbb{R}_{+}^{B} \backslash E$. The sets $E, F$ are not generally intervals, but are elementary sets which are restricted only in the finite set of dimensions $M$. Let $E(M), F(M)$ denote the projections of $E, F$ into the finite-dimensional space $\mathbb{R}_{+}^{M}$. Then, using the finite additivity in $\mathbb{R}_{+}^{M}$ of the integrals $Q(I(M))$ and a finite-dimensional version of Fubini's theorem, we have $(\mathcal{E}) \sum G(x, N, I[N])=\int_{E(M)} Q(I(M)) d y(M)$ and

$$
(\mathcal{E}) \sum G(x, N, I)+\int_{F(M)} Q(I(M)) d y(M)=\int_{\mathbf{R}_{+}^{M}} Q(I(M)) d y(M)=1,
$$

so if we can choose a gauge $\gamma_{2}$ so that the supplementary term has value less than $\varepsilon$, that is,

$$
\int_{F(M)} Q(I(M)) d y(M)<\varepsilon
$$

then we have the required result. We can certainly find $U_{j}, V_{j}, j=1, \ldots, m$, so that $J(M)=\left[U_{1}, V_{1}\left[\times \cdots \times\left[U_{m}, V_{m}[\right.\right.\right.$ and $J[M]$ are, respectively, an interval of $\mathbb{R}_{+}^{M}$ and the corresponding cylindrical interval, with $J(M) \subset$ $E(M) \subset \mathbb{R}_{+}^{M}$. In fact $J(M)$ is a compact rectangular interval of $\mathbb{R}_{+}^{M}$, and $E(M)$ consists of $J(M)$ combined with a finite number of other rectangular intervals, some without upper or without lower bounds in some dimensions of $\mathbb{R}_{+}^{M}$. We then have

$$
\int_{J(M)} Q(I(M)) d y(M) \leq(\mathcal{E}) \sum G(x, N, I)<\int_{\mathbb{R}^{M}} Q(I(M)) d y(M)=1 .
$$


For $j=1, \ldots, n$ we can take $U_{j}$ and $V_{j}$ to be, respectively, very small and very large positive numbers satisfying

$$
\begin{aligned}
& U_{j}=\max \left\{\delta_{t}(x(t)):(x, I[N]) \in \mathcal{E}, x(t)=0 \text { for some } t \in N\right\}, \\
& V_{j}=\max \left\{\left(\delta_{t}(x(t))\right)^{-1}:(x, I[N]) \in \mathcal{E}, x(t)=\infty \text { for some } t \in N\right\},
\end{aligned}
$$

If it could be shown that $\int_{\mathbb{R}^{M}} Q(I(M)) d y(M)-\int_{J(M)} Q(I(M)) d y(M)<\varepsilon$, we would have the result we require. However, following a comment by V.A. Skvortsov, while we can certainly choose $\delta_{t}(x(t))$ to be arbitrarily small for the critical points $x(t)$ for which $x(t)$ equals 0 or $\infty$ with $t \in N$, this is not sufficient to give us the latter inequality, since the coordinates $y_{j}$ of $y(M)$ are scaled, not by $t_{j}$ but by the differences $t_{j}-t_{j-1}$.

However, we may proceed as follows. It is shown in [7] that, with $M_{0}$ a given finite set of dimensions $t$, the Wiener measure $w$ has variation 0 in the set $D_{M_{0}}$ of $x$ in $\mathbb{R}^{[0,1]}$ which are not continuous at some $t \in M_{0}$. When the so-called "continuous modification" of $w$ is constructed, by an argument described in $[9,10]$, and, using the same symbol for the modified $w$, we find that $w$ has variation zero in the set of $x$ which are discontinuous at any $t \in[0,1]$.

A similar argument shows that (a "continuously modified") $G$ has variation zero in the set $D_{+}$of $x \in \mathbb{R}_{+}^{B}$ which are discontinuous at some $t \in B$. So if $X$ is any subset of $D_{+}$, a gauge $\gamma$ can be chosen so that

$$
\left(\mathcal{E}_{\gamma}\right) \sum G(x, N, I[N]) \mathbf{1}_{X}(x)<\varepsilon .
$$

Take $X$ to be those $x \in \mathbb{R}_{+}^{B}$ for which $x(t)$ equals 0 or $\infty$ for some $t$, so $X$ is a subset of $D_{+}$. Then (10) gives (9), and we have the result.

The complication in the above proof arises when terms $h(x, N, I)$ in the Riemann sum having $x(t)=0$ or $\infty$ for $t \in N$ are omitted. But it is only necessary to omit such terms when the corresponding integrand value is undefined. This is not the case for the integrand $G(x, N, I[N])$, since, by (4), this is $Q(I(N))$, which is defined in these cases, by means of a finite dimensional integral.

Therefore the above proof shows that $G$ is integrable whether we use gauges $\gamma_{1}$ or $\gamma_{2}$, and we have

Proposition 4. $G(x, N, I[N])$ is integrable in $\mathbb{R}_{+}^{B}$ and ${ }^{1} \int G(x, N, I[N])=1$. 


\section{A problem}

If we could show that $g(x, N, I)$ is variationally equivalent to $G(x, N, I)$, we could then deduce that $g(x, N, I)$ is integrable in $\mathbb{R}_{+}^{B}$, with integral 1 .

To prove the variational equivalence, we might reason as follows. Given $x \in \overline{\mathbb{R}}_{+}^{B}$, for each finite $N$ choose a gauge $\gamma$ so that

$$
\left|\prod_{j=1}^{n} g_{j}\left(x_{j}, x_{j-1}\right)-\prod_{j=1}^{n} g_{j}\left(y_{j}, y_{j-1}\right)\right|<\frac{\varepsilon}{2} \prod_{j=1}^{n} g_{j}\left(x_{j}, x_{j-1}\right) ;
$$

and

$$
\prod_{j=1}^{n} g_{j}\left(y_{j}, y_{j-1}\right)>\frac{1}{2} \prod_{j=1}^{n} g_{j}\left(x_{j}, x_{j-1}\right)
$$

We would then get

$$
\begin{aligned}
& |g(x, N, I)-G(x, N, I)|=|q(I(N))-Q(I(N))| \\
& =\left|\int_{I(N)}\left[\prod_{j=1}^{n} g_{j}\left(x_{j}, x_{j-1}\right)-\prod_{j=1}^{n} g_{j}\left(y_{j}, y_{j-1}\right)\right] d y(N)\right| \\
& \leq \int_{I(N)}\left|\prod_{j=1}^{n} g_{j}\left(x_{j}, x_{j-1}\right)-\prod_{j=1}^{n} g_{j}\left(y_{j}, y_{j-1}\right)\right| d y(N) \\
& \leq \varepsilon / 2 \int_{I(N)} \prod_{j=1}^{n} g_{j}\left(x_{j}, x_{j-1}\right) d y(N) \\
& \leq \varepsilon \int_{I(N)} g_{j}\left(y_{j}, y_{j-1}\right) d y(N)=\varepsilon G(x, N, I) .
\end{aligned}
$$

Finally we would choose $\gamma$ so that for all $\mathcal{E}_{\gamma}$,

$$
\left(\mathcal{E}_{\gamma}\right) \sum|g(x, N, I)-G(x, N, I)| \leq \varepsilon\left(\mathcal{E}_{\gamma}\right) \sum G(x, N, I)<2 \varepsilon .
$$

This would give variational equivalence of $G$ and $g$. However, step (11) seems to require a gauge of the form $\gamma_{3}$, and there is no proof that $\gamma_{3}$-fine divisions of $\mathbb{R}_{+}^{B}$ exist.

The argument above was given originally by Henstock in [3]. What are the significance and interest of proving the variational equivalence of $G$ and $g$, and what are the main ideas involved? 
If we were to collapse $B$ down to a single dimension (so $B$ consists of a single element, and consider a related Gaussian integral

$$
F(I)=\int_{I} f(y) d y \text { where } f(y)=\exp \left[-(\ln y)^{2}\right] \text { and } I=[u, v[,
$$

we see that the indefinite integral $F(I)$ is a primitive function for $f(y)$. This idea can be developed into a connection between $f(y)|I|$ and $F(I)$, which are variationally equivalent by the Saks-Henstock Lemma [7] (page 28). In $\mathbb{R}_{+}^{B}$ the functions $g(x, N, I)$ and $G(x, N, I)$ have an analogous relationship. This would be a useful analytic connection to have at our disposal in the study of Black-Scholes and similar integrands.

And what about the approach to a proof described above? We can see by the continuity of the integrals involved, that $f(y)|I|, F(I)$ in one dimension, and $g(x, N, I), G(x, N, I)$ in infinite dimensions, can (respectively) be made arbitrarily close in value by taking the $I$ small enough. So it might come as no great surprise if, when either expression (of each pair) is integrated that the result might be the same as integrating the alternate expression. Besides this intuition, what else might be needed?

If we were to consider the variational equivalence of $f(y)|I|$ and $F(I)$ in a compact interval $[a, b]$, we could choose a gauge to ensure that

$$
\left|f(y)-\frac{F(I)}{|I|}\right|<\frac{\varepsilon}{b-a}, \quad|f(y)| I|-F(I)|<\varepsilon \frac{|I|}{b-a} .
$$

Then Riemann sums $\sum|f(y)| I|-F(I)|$ would be less than $\varepsilon$. To prove variational equivalence of these functions in $[0, \infty[$, we would need a gauge on $[0, \infty[$ so that

$$
|f(y)| I|-F(I)|<\varepsilon h(y, I)
$$

where $h(y, I)>0$ is integrable (or at least has generalised bounded variation) in $\left[0, \infty\left[\right.\right.$. For instance, $h(y, I)=\exp \left[-y^{2}\right]|I|$ would be all right.

When we examine Riemann sums

$$
\sum|g(x, N, I)-G(x, N, I)| \text {, or } \sum \mid q(I(M)-Q(I(M)) \mid,
$$

the number of terms in the sum will generally be extremely large, and obtaining an arbitrarily small result is a much more delicate task. Suppose, for instance, that we have a gauge in $\mathbb{R}_{+}^{B}$ so that, for each $t \in M$, the range of $x(t)$ is divided into ten subintervals, and suppose that the gauge causes the insertion of nine partition points $t_{j}$ in $\left.\left.B=\right] t_{0}, T\right]$. Such a gauge is relatively coarse. Even so, the Riemann sum will have up to ten billion terms. And, even if we manage to make the errors or differences

$$
\left|g_{j}\left(x_{j}, x_{j-1}\right)\right| I_{j}\left|-\int_{u_{j}}^{v_{j}} g_{j}\left(y_{j}, y_{j-1}\right) d y_{j}\right|
$$


very small, unless we manage things very carefully the Riemann sum may result in the accumulated errors being arbitrarily large rather than arbitrarily small.

The trick lies in finding a suitable function corresponding to $h(y, I)$ of (13). We have such functions at hand. We used $g(x, N, I)$ itself for this part of the argument, and then, because of (12), we were able to substitute $G(x, N, I)$ which we know to be integrable from Proposition 3. These steps would require us to be able to choose gauges $\delta_{N}$ for each $N$, suggesting that $\gamma_{3}$ or $\gamma_{5}$ might be the appropriate kinds of gauge to obtain a proof.

\title{
References
}

[1] Elliott, R. J., Kopp, P. E., Mathematics of Financial Markets, Springer-Verlag, New York, 1999.

[2] Henstock, R., Linear Analysis, Butterworths, London, 1968.

[3] Henstock, R., Integration in product spaces, including Wiener and Feynman integration, Proc. London Math. Soc. 27 (1973), 317-344.

[4] Henstock, R., The General Theory of Integration, Clarendon Press, Oxford, 1991.

[5] Henstock, R., The construction of path integrals, Math. Japon. 39(1) (1994), 15-18.

[6] Kwok, Y. K., Mathematical Models of Financial Derivatives, Springer, Singapore, 1998.

[7] Muldowney, P., A General Theory of Integration in Function Spaces, Pitman Res. Notes Math. Ser. 153, Longman, Harlow, 1987.

[8] Muldowney, P., Introduction to Feynman integration, J. Math. Study 27(1) (1994), 127-132.

[9] Muldowney, P., Topics in probability using generalised Riemann integration, Proc. Royal Irish Acad. Sect. A 99(1) (1999), 39-50.

[10] Muldowney, P., Feynman's path integrals and Henstock's non-absolute integration, J. Appl. Anal. 6(1) (2000), 1-24.

[11] Muldowney, P., The Henstock integral and the Black-Scholes theory of derivative asset pricing, Real Anal. Exchange 26(1) (2000-2001), 117-132.

\author{
P. Muldowney \\ Magee College \\ UNIVERSITY OF ULSTER \\ NORTHLAND ROAD \\ DERRY BT48 7JL \\ NORTHERN IRELAND \\ P.MULDOWNEY@ULSTER.AC.UK
}

